
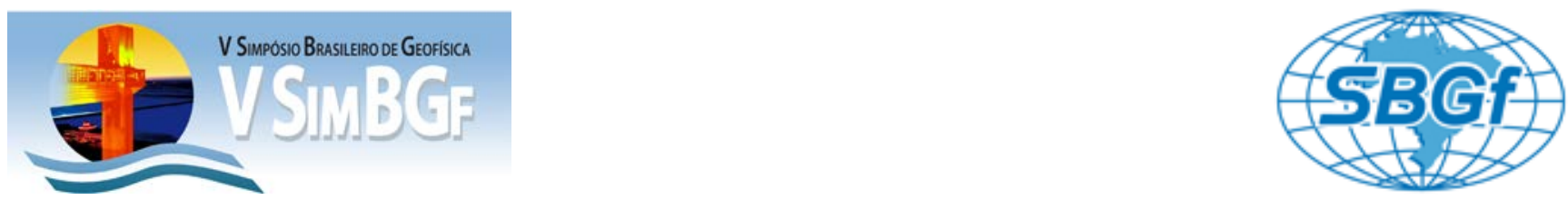

\title{
Increasing the CSEM application window; Extracting regional structures
}

Lars Lorenz*, EMGS do Brasil and Håkon T. Pedersen, EMGS Americas

Copyright 2012, SBGf - Sociedade Brasileira de Geofísica

Este texto foi preparado para a apresentação no V Simpósio Brasileiro de Geofísica, Salvador, 27 a 29 de novembro de 2012. Seu conteúdo foi revisado pelo Comitê Técnico do V SimBGf, mas não necessariamente representa a opinião da SBGf ou de seus associados. É proibida a reprodução total ou parcial deste material para propósitos comerciais sem prévia autorização da SBGf.

\section{Abstract}

CSEM is generally perceived as being a low-resolution method used exclusively for hydrocarbon detection. In this paper, we introduce a methodology which has proven to be robust for structural extraction using wide-azimuth 3D CSEM data. By tracking variations in the electrical anisotropy of sediments and by utilizing imaging attributes, we show that large scale structural information can be reconstructed and can be used where seismic data coverage is sparse. Through an iterative process, the end product will be a structurally guided resistivity cube.

\section{Introduction}

The Controlled Source Electromagnetic (CSEM) method was introduced by Eidesmo et al. (2002) and Ellingsrud et al. (2002) as a remote sensing application for hydrocarbon exploration. An extensive overview of the methodology is provided by Constable and Srnka (2007), but in short the method is used for mapping the resistivity of the subsurface. The relationship between hydrocarbon saturation and resistivity was introduced by Archie (1942) and exhibits an exponential resistivity change for increasing hydrocarbon saturations, clearly separating non-commercial from commercial saturations.

In the last 10 years, the method has undergone a rapid development. Acquisition expanded from a 2D line across a single prospect to larger 2D CSEM campaigns for portfolio ranking to currently large scale regional 3D CSEM surveys and 3D campaigns for portfolio ranking with the acquisition of wide-azimuth CSEM data. On the processing side, the introduction of 3D inversion and the recognition of resistivity anisotropy as an important factor for reliable inversion and interpretation increased the confidence in the CSEM results significantly (Bekker and Danielsen, 2011). Not only did this allow for a significant expansion of the application window to more geologically complex areas, but also to expand it beyond the initial pure-play exploration focus into the appraisal domain as e.g. demonstrated by Morten et al. (2012).

\section{Motivation}

CSEM is generally perceived as being a low-resolution method, providing very limited to no structural information, and to rely heavily on seismic for this type of information in the inversion and interpretation stage. This poses a quandrum for the application in frontier areas with only sparse 2D seismic coverage like the Equatorial Margins of Brazil. Large seismic data holes of $100 \mathrm{sq} . \mathrm{km}$ will be prone to interpolation artifacts and thereby imposing a wrongfully bias to any structurally guided CSEM inversion.

A review of the world-wide portfolio of wide-azimuth 3D CSEM surveys revealed some astounding similarties across a range of geographical areas. Figure 1 shows one of the comparisons with very similar character in the resistivity anisotropy for data acquired offshore Malaysia (from: Ganesan et al., 2012) and offshore Brazil.
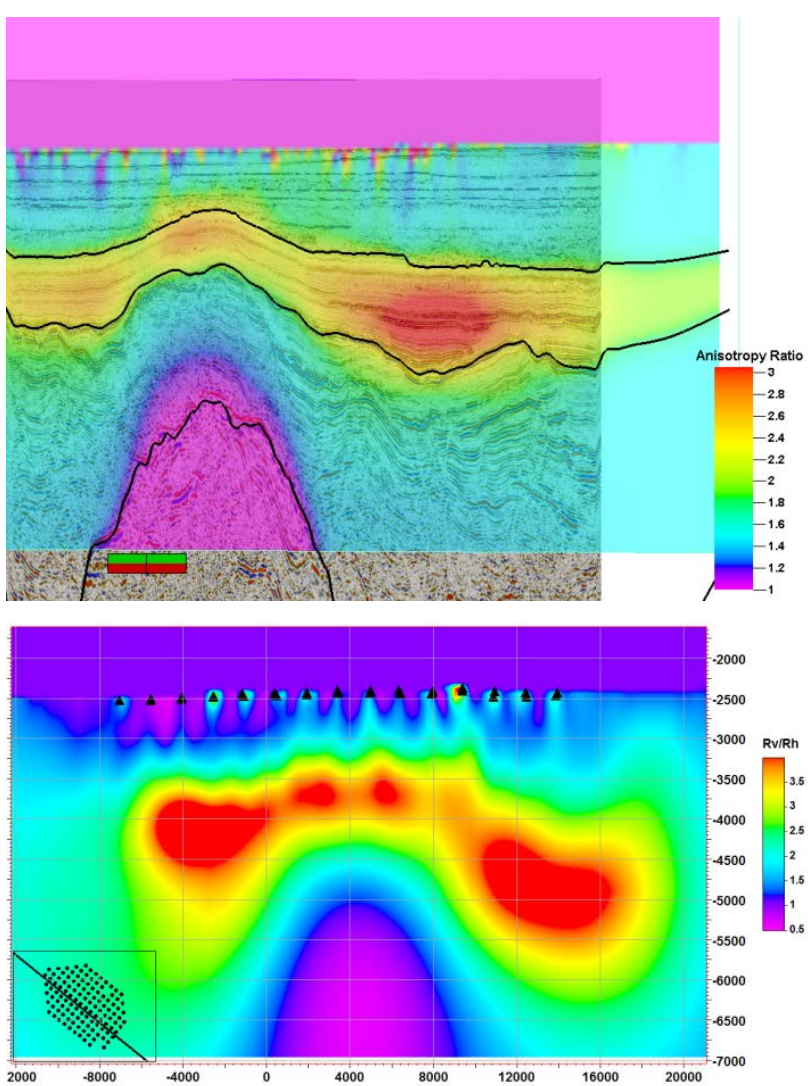

Figure 1: Resistivity anisotropy ratio for surveys in offshore Malaysia (top) and offshore Brazil (bottom) exhibiting very similar character.

If considering that the basic play elements for these areas are similar, the similarities may not occur so strange from a geological perspective, though. Basic elements of regional seal (shale), as well as underlying metamorphic/basement rocks may be expected to exhibit some comparable properties. If so, additional geological information could be gained from the CSEM data in 
excess of the sole resistivity cube. This will allow to perform structurally guided inversions even in the absence or sparse samling of seismic data. It will also provide an additional QC tool when using seismic data as acoustic property contrast do not need to reflect electric property contrasts, potentially resulting in the use of inappropriate structural information in a guided CSEM inversion.

\section{Methodology}

The primary aim of the developed methodology is to map interfaces for the change of anisotropy factors at a regional scale. These changes are primarily associated with shale layers, exhibiting significant resistivity anisotropy as documented by Ellis et al. (2010), and represent an important element in oil and gas exploration due to their sealing capability. For this methodology development, we are using the 3D inversion code which is described by Zach et al. (2008) and Stoeren et al. (2008).

As we intend to use the anisotropy behaviour as an indicator for layer boundaries, it is required that the start model is either isotropic, or exhibits a constant anisotropy ratio to reduce the bias in the update of the anisotropy. The resulting anisotropy cube can then be analyzed for changes in the anisotropy ratio which can be mapped out. This works best in areas with low lithology complexity. If thin resistors, either in form of hydrocarbons, carbonates, volcanics or locally deposited calcareous shale are present, the anisotropy ratio can still be a rather complicated attribute to deal with. Figure 2 outlines the high variability of the anisotropy for an area from NE Brazil.

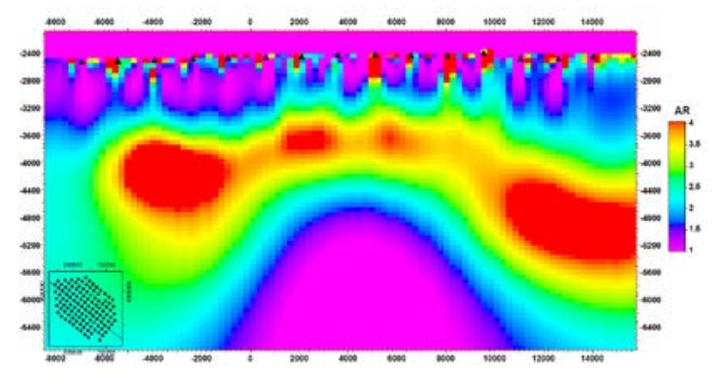

Figure 2: Anisotropy ratio for an inversion result in $\mathrm{NE}$ Brazil, exhibiting some resemblance of structure, but also a significant variability in the central higher anisotropic band.

One of the reasons for the additional complications is that the sensitivity to thin resistors is mainly restricted to the vertical resistivity cube. Also, thin resistors have a tendency to be smeared out in thickness. The anisotropy ratio will result in an overprint of the sharp boundary of the background lithology by the smeared out expression of the thin resistor in the vertical resistivity. The other reason is the dominance of receiver footprint artifacts in the vertical resistivity, challenging our ability to map out distinct features in the shallower subsurface.

Instead of attempting to mitigate these behaviours by use of regularization, and thereby biasing the inversion, we decided to look at the horizontal resistivity cube. As high resistive thin geobodies are primarily mapped into the vertical resistivity cube, the horizontal resistivity exhibits much less of these distortions (Morten et al., 2010). The resulting horzontal resistivity cube is now normalized by the initial starting model, resulting in an attribute which we called for reasons of lacking imagination Normalized Horizontal Update (NHU).

By using constant anisotropy ratio in the start model, we introduce an error as sediments tend to have a large variation of anisotropy. By tracking the change between final and initial horizontal resistivity model, we highlight the areas where this error in assumption is most pronounced and the inversion corrections are strongest. A shale layer would be the prime candidate for such a correction, and quasi-isotropic features as basement structures would exhibit such an effect as well, just in opposite direction. Extracting the NHU for the same NE Brazil data set, shown in Figure 3, underlines the more defined character of the boundaries. These boundaries can now be mapped and incorporated into a more refined start model.

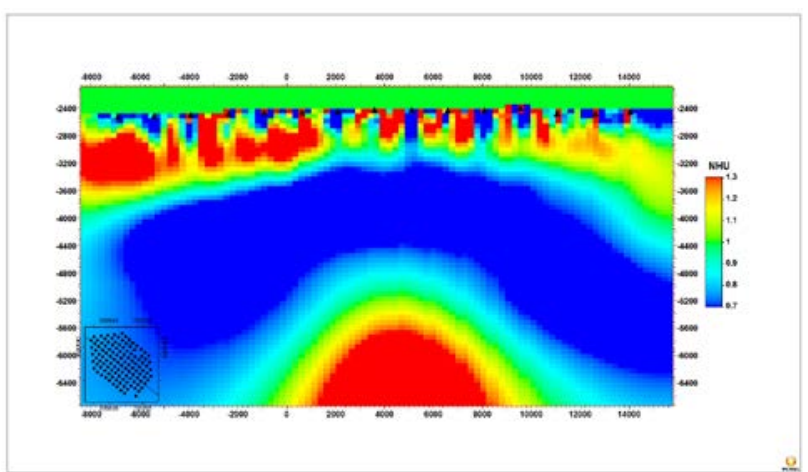

Figure 3: Normalized Horizontal Update of inversion result versus start model exhibiting a more defined structural character. A value below 1 means that the model updates are a reduction in horizontal resistivity.

\section{Discussion}

By including the derived boundaries in the final inversion start model, a much better continuity of the regional resistive events was achieved. This result was not too surprising as CSEM inversions tend to have a bias towards the initial start model. To verify if the results were geologically meaningful, we analyzed seven different 3D CSEM data sets, located in Asia, West Africa and GOM with different structural complexity. For areas with higher complexity, it proved that the results significantly improved when only using wide-azimuth data, further subduing the effects of local thin resistive events. One of the observations of the verification process was that 3D data sets with limited lateral extension and resulting narrow 
azimuth coverage showed significant deviations towards the edge of the survey area due to under-sampling and the results were significantly less reliable in these areas.

As the verification required proprietary depth converted 3D seismic with interpretation of the major lithology boundaries, an anonymous statistical analysis for two horizons of a $300+$ square-kilometre survey in the GOM is shown.

Figure 4 shows some statistics for a shallow lithology interface $(\sim 400 \mathrm{mbsf})$ and for a deeper shale layer $(\sim$ 1500 mbsf). The median deviation is for both data sets approximately $60 \mathrm{~m}$. This is within the size of a typical inversion grid cell for a regular finite difference inversion grid. The large deviations in relation to burial depth for the shallow layer occur to be related to the CSEM event and seismic stratigraphic event deviating consistently for one part of the survey area. A potential explanation is that the stratigraphic event is not identical to the lithology boundary as mapped by the CSEM event.

For the deeper layer, the error as a fraction of the burial depth clusters in the range of $6 \%$ to $11 \%$ which is not worse than what can be expected from an uncalibrated 2D seismic data set. This supports that this methodology can support the interpretation and processing of CSEM data in frontier areas by obtaining structural information in depth in a frontier area within acceptable bounds.
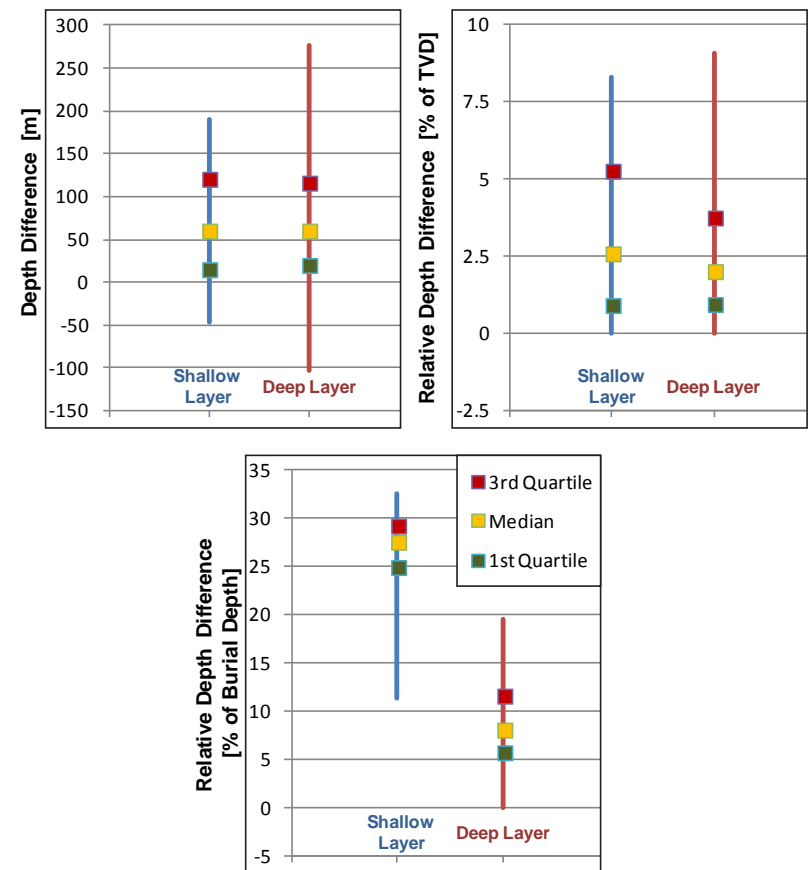

Figure 4: Overview on the deviation between the CSEM derived horizons and seismic derived horizons for a 300+ sqkm 3D data set. Three measures are provided, absolute difference (upper left), relative difference to TVD (upper right), and relative difference to burial depth (bottom)
In addition, this methodology may open up the possibility to use structural information from CSEM data for early stage basin models when only 2D seismic data is available, but also to provide an additional guide to identify events in the intepretation of CSEM data which cause resistivity trends. Considering that structural as well as qualitative information in form of anisotropy ratios are derived, it may even aid seismic interpreters in the regional assessment of the general stratigraphy in an area, even though a reference data base on rock properties with respect to resistivity anisotropy may be needed to achieve the required level of confidence.

Also, at later stages of the exploration workflow, these structural information could provide helpful guidelines for a refinement of the interpretation and lithology distribution as they provide an additional data set which can supplement well logs and may be able to provide answers to questions which are not conclusively resolved by seismic, e.g. presence of basement versus older and more compacted sediments.

\section{Conclusion}

We developed a workflow to extract structural information from a 3D wide-azimuth CSEM data set by analyzing resistivity attributes. Using these structural information resulted in more consistent inversion results. We tested this workflow against a range of these CSEM data sets from different basins. The wide-azimuth component of the data sets was especially important in areas of high structural or lithological complexity as they allowed for the necessary level of confidence to use the structural information.

At the current stage, the workflow was mainly employed to identify the boundaries of shale layers which have a very distinct anisotropy character. Overall, the workflow proved to be robust and the depth estimates were in line with the estimates which were derived from the depth converted seismic. This suggests that the results of a CSEM inversion, even though the method being very low frequent, contains valuable structural information. We think that this workflow makes the use of CSEM more versatile even in areas with very limited seismic coverage and could also provide additional information for seismic interpretation and processing.

We have shown that structure can be inferred from careful inversion of wide azimuth CSEM data. This method has certain limitations to depth of penetration, and the next step in the use of EM methods to develop deeper structural models will be the careful integration, and possibly joint inversion, of CSEM and magneto telluric data. This would allow to provide information on deep structural controls for frontier basins which are an important input for the regional assessment of prospectivity. 


\section{Recognition}

We like to thank EMGS for the permission to publish this work.

\section{References}

Eidesmo, T., S. Ellingsrud, L. M. MacGregor, S. Constable, M. C. Sinha, S. Johansen, F. N. Kong, and H.Westerdahl, 2002. Sea bed logging (SBL), a new method for remote and direct identification of hydrocarbon filled layers in deepwater areas, First Break, 20: 144-152

Ellingsrud, S., Sinha, M.C., Constable, S., MacGregor, L.M., Eidesmo, T. \& Johansen, S., 2002. Remote sensing of hydrocarbon layers by Sea Bed Logging (SBL): results from a cruise offshore Angola, The Leading Edge, 21: 972-982

Constable, S. \& Srnka, L. J., 2007. An introduction to marine controlled-source electromagnetic methods for hydrocarbon exploration, Geophysics, 72: 3-12

Archie, G.E., 1942. The electrical resistivity log as an aid in determining some reservoir characteristics, Petroleum Transactions of AIME, 146: 54-62

Bekker, R. \& Danielsen, J., 2011. The future of marine CSEM, First Break, 29: 77-81

Morten, J. P., Roth, F., Karlsen, S. A., Timko, D., Pacurar, C., Olsen, P.A., Nguyen, A.K., Gjengedal, J., 2012. Field appraisal and accurate resource estimation from 3D quantitative interpretation of seismic and CSEM data, The Leading Edge, 31: 447-456

Ganesan, V., Pedersen, H. T., Chandola, S. K., 2012. Pitfalls in CSEM inversion; a case study of a false positive, Expanded Abstract, Petroleum Geoscience Conference \& Exhibition, Kuala Lumpur, Malaysia

Ellis, M. H., Sinha, M., Parr, R., 2010. Role of fine-scale layering and grain alignment in the electrical anisotropy of marine sediments, First Break, 28: 49-57.

Zach, J. J., Bjoerke, A. K., Stoeren, T., Maaoe, F. A., 2008. 3D inversion of marine CSEM data using a fast finite-difference time domain forward code and approximate Hessian-based optimization, 78th SEG Annual International Meeting, Las Vegas, USA, Expanded Abstracts, 614-618

Stoeren, T., Zach, J. J., Maaoe, F. A., 2008. Gradient Calculations for 3D Inversion of CSEM data using a fast finite-difference time-domain modelling code, 70th EAGE Conference \& Exhibition, Rome, Italy, Expanded Abstracts, P104

Morten, J.P., Bjoerke, A.K., Nguyen, A.K, 2010. Hydrocarbon reservoir thickness resolution in 3D CSEM anisotropic inversion, 80th SEG Annual International Meeting, Denver, USA, Expanded Abstracts, 599-603 